$\begin{array}{cl}\begin{array}{c}\text { Revue } \\ \text { de Ihistoire }\end{array} & \text { Revue de l'histoire des religions } \\ \text { des religions } & 2 \mid 2010 \\ & \text { Varia }\end{array}$

\title{
Le « jeu rituel » du noaidi chez les Samis préchrétiens (Laponie, XVII ${ }^{\mathrm{e}}-\mathrm{XVIII}{ }^{\mathrm{e}}$ siècles)
}

The " ritual play " of the noaidi among the pre-Christian Sami (Lapland, 17th18th centuries)

\section{Håkan Rydving}

\section{(2) OpenEdition}

\section{Journals}

Édition électronique

URL : http://journals.openedition.org/rhr/7577

DOI : $10.4000 /$ rhr.7577

ISSN : 2105-2573

Éditeur

Armand Colin

Édition imprimée

Date de publication : 1 juin 2010

Pagination : 211-230

ISBN : 978-2200-92656-4

ISSN : 0035-1423

Référence électronique

Håkan Rydving, "Le « jeu rituel » du noaidi chez les Samis préchrétiens (Laponie, XVII -XVIII siècles) », Revue de l'histoire des religions [En ligne], 2 | 2010, mis en ligne le 01 juin 2013, consulté le 30 avril 2019. URL : http://journals.openedition.org/rhr/7577 ; DOI : 10.4000/rhr.7577 


\section{Le « jeu rituel » du noaidi chez les Samis préchrétiens (Laponie, $\mathrm{XVII}^{\mathrm{e}}-\mathrm{XVIII}{ }^{\mathrm{e}}$ siècles)}

L'article traite certains problèmes que posent les sources de l'anthropologie historique, notamment celles qui concernent la religion des Samis (Lapons) aux XVII et XVIII siècles. Pour le noaidi, le personnage religieux le plus important chez les Samis préchrétiens, les représentations rituelles avaient souvent un caractère érotique. Par exemple, à l'aide de gestes et de sons, le noaidi essayait d'imiter l'accouplement des animaux. Or les textes des missionnaires protestants - qui constituent nos principales sources - sont (ou semblent) presque totalement muets à ce sujet. Les chercheurs restent également silencieux. En s'inspirant des travaux de Roberte Hamayon, l'auteur se propose donc de relire les sources, d'analyser de nouveau les vocabulaires samis et de reconstruire l'image des " jeux rituels ».

\section{The « ritual play » of the noaidi among the pre-Christian Sami (Lapland, 17th -18 th centuries)}

The article treats certain problems posed by the sources of historical anthropology, notably those that concern the religion of the Sami (Lapps) during the 17th and 18th centuries. For the noaidi, the most important religious figure among the pre-Christian Sami, the ritual performances often had an erotic character. For example, the noaidi tried to immitate the pairing of animals with the help of gestures and sounds. Yet, the texts of the protestant missionaries - that constitute our main sources - are (or seem to be) nearly totally silent on this topic. Researchers remain silent, as well. Inspired by Roberte Hamayon's studies, the author proposes to read the sources anew, to analyse the Sami vocabularies over again and to reconstruct the image of the " ritual plays ". 


\section{INTRODUCTION}

La lecture des textes d'autres chercheurs peut nous amener à voir notre propre matériel sous un jour différent. Cela concerne tout particulièrement les textes portant sur d'autres contextes culturels que ceux que l'on étudie soi-même et qui adoptent une autre démarche que la nôtre. Nous nous apercevons alors brusquement que, pour avoir repris certaines idées sans réflexion, nous avons mal interprété ou négligé des informations capitales que contiennent les sources. Certains chercheurs possèdent la faculté particulière de nous faire mettre le doigt sur nos conceptions irréfléchies et sur nos négligences. Ils nous forcent à entreprendre un travail de déconstruction et de reconstruction méthodologique et théorique.

Pour illustrer la manière dont la révision des bases théoriques peut nous amener à considérer ce que nous ne pouvions voir auparavant, je montrerai comment je suis moi-même revenu sur ma compréhension d'un mot sami (lapon) appartenant à la terminologie des rituels. Dans un article publié en 1987, j'avais inventorié et analysé les terminologies relatives aux rites du spécialiste des rituels, le noaidi ${ }^{1}$. L'un des mots que je cite dans cet article est le verbe gihkat qui désigne à la fois le cri que certains oiseaux (notamment le grand coq de bruyère) poussent pendant l'accouplement et le bruit que fait le noaidi en accomplissant certains rites; mais ce que j'avais retenu à propos de l'oiseau, ce n'était pas son cri mais le fait qu'il devient aveugle et sourd pendant une partie de l'accouplement, ce que je traduisais par «un état d'extase». Prisonnier de la théorie de l'« extase » comme trait caractérisant le « chamanisme », je croyais que le terme avait été choisi parce que l'oiseau était considéré comme étant en « extase », exactement comme le noaidi ( « chamane »). C'est pourquoi je n'avais pas vu ce qui est aujourd'hui évident pour moi, après la lecture des articles dans lesquels Roberte Hamayon déconstruit le concept d'« extase » : en l'occurrence qu'il existe une analogie significative entre le cri du

1. H. Rydving, "Shamanistic and postshamanistic terminologies in Saami », dans T. Ahlbäck (éd.), Saami Religion, Stockholm, Almqvist \& Wiksell International, 1987, p. 185-207. 
noaidi pendant le rite et le cri du grand coq de bruyère pendant l'accouplement. L'information était là; je l'avais même presque notée dans l'article, mais je n'en voyais pas le sens parce que mon point de départ théorique me faisait rechercher autre chose : des exemples d'« extase ». Ce cas personnel illustre bien comment les grilles théoriques de nos recherches déterminent dans une large mesure notre vision; il nous rappelle aussi à quel point il est important d'en avoir conscience et de les énoncer dans ses textes.

Ayant déconstruit le concept d' « extase $^{2} »$, Hamayon recourt au concept de « jeu» pour reconstruire les descriptions des religions indigènes de la Sibérie ${ }^{3}$. C'est ce concept, notamment la dimension érotique des rituels, qui constituera mon point de départ pour une nouvelle lecture des sources de la religion sami et la reconstruction de la conduite rituelle du noaidi.

Je reconnais volontiers qu'avant de lire les articles d'Hamayon sur ce thème, j'employais le mot « extase » comme concept analytique dans mon étude du noaidi chez les Samis. Aujourd'hui par contre, je vois que mes sources concernent en partie des éléments qui diffèrent de ceux que j'avais vus à travers l'approche théorique à laquelle j'adhérais à l'époque.

\section{LES SOURCES}

Tournons-nous vers l'exemple sami et voyons ce qu'une relecture des sources à la lumière du concept de «jeu », et notam-

2. R. N. Hamayon, « Pour en finir avec la « transe » et l' « extase » dans l'étude du chamanisme $»$, Études mongoles et sibériennes $n^{\circ} 26,1995$, p. 155-190.

3. Voir R. N. Hamayon, «Stakes of the game, Life and death in Siberian shamanism », Diogène : revue internationale de sciences humaines $\mathrm{n}^{\circ} 158,1992$, p. $69-85$; R. N. Hamayon, « Pourquoi les « jeux » plaisent aux esprits et déplaisent à Dieu. Le « jeu », forme élémentaire de rituel à partir d'exemples chamaniques sibériens », dans G. Thinès et L. de Heusch (éd.), Rites et ritualisation, Paris, Librairie philosophique J. Vrin, 1995, p. 65-100; R. N. Hamayon, "L'épingle du jeu », La Revue du MAUSS n ${ }^{\circ} 12,1998$, p. 103-121 ; R. N. Hamayon, " Des usages de « jeu » dans le vocabulaire rituel du monde altaïque », Études mongoles et sibériennes $\mathrm{n}^{\circ}$ 30-31, 2000, p. 11-45. Sur «le jeu», voir J. Huizinga, Homo ludens, Essai sur la fonction sociale du jeu, Paris, Gallimard, (1938) 1988; R. Caillois, Les Jeux et les Hommes, Paris, Gallimard, (1958) 2000; G. Bateson, Steps to an Ecology of Mind, New York, Ballantine, 1972; S. D. Gill, « Play », dans W. Braun et R. T. McCutcheon (éd.), Guide to the Study of Religion, Londres, Cassell, 2000, p. $451-462$. 
ment des aspects que l'on pourrait caractériser comme « jeu érotique », peut nous apporter dans la compréhension de la religion préchrétienne.

Avant de nous lancer dans cette entreprise, il faut au préalable répondre à une question fondamentale: d'où proviennent nos connaissances sur la religion préchrétienne des Samis aux XVII et $\mathrm{XVIII}$ e siècles?

Bien que certains éléments en aient été longtemps conservés dans les croyances populaires, il faut bien reconnaître que la majeure partie de ce qui faisait la religion sami a disparu au XVIII ${ }^{e}$ siècle après plusieurs siècles d'une activité missionnaire qui culmina à partir de la fin du XvII ${ }^{\mathrm{e}}$ siècle. Or c'est de cette période - qui constitue la ligne de faille entre l'ancienne et la nouvelle religion - que date la majeure partie de nos sources. On peut considérer certaines d'entre elles comme primaires, en ce sens qu'elles proviennent des croyants eux-mêmes, c'est-à-dire des Samis qui continuaient de vivre selon les normes de la religion indigène. Mais le problème que posent ces sources (les figures difficilement interprétables dessinées sur les tambours ainsi que différents types de lieux sacrés, de lieux sacrificiels et de tombes), c'est que, comme toutes les sources non verbales, elles doivent être interprétées à partir des informations que nous recueillons dans les textes. Nous sommes ainsi obligés d'avoir recours aux sources secondaires écrites, ne serait-ce que pour comprendre le reste du matériel, une situation qui met en évidence l'importance des sources verbales.

Ces dernières se divisent en plusieurs catégories ${ }^{4}$ :

1) quelques textes antérieurs au XVII siècle;

2) les récits des voyageurs, notamment français et italiens, qui ont visité l'Europe septentrionale aux XVII et XVIII ${ }^{\mathrm{e}}$ siècles;

3) les procès-verbaux des interrogatoires menés par les tribunaux pour juger les Samis soupçonnés de ce que l'on appelait « la pratique de la sorcellerie »;

4) différentes archives laissées par les missionnaires;

4. Voir à ce sujet H. Rydving, Samisk religionshistoria, Några källkritiska problem, Stockholm, Almqvist \& Wiksell International, 1995; H. Rydving, " The missionary accounts from the 17th and 18th centuries, The evaluation and interpretation of the sources », dans J. Pentikäinen (éd.), Sami Folkloristics, Turku, Nordic Network of Folklore, 2000, p. 17-39. 
5) l'important matériel traditionnel enregistré depuis la fin du $\mathrm{XIX}^{\mathrm{e}}$ jusqu'au début du $\mathrm{XX}^{\mathrm{e}}$ siècle, que l'on trouve dans les collections d'archives et de livres concernant les coutumes des Samis;

6) les terminologies religieuses;

7) et, enfin, les noms de lieux sacrés.

Ces types de sources ne constituent cependant que des compléments aux sources (8) que l'on appelle d'habitude tout simplement «les sources écrites»: un ensemble abondant et disparate de textes rédigés vers la fin du XVII ${ }^{\mathrm{e}}$ siècle et le début du XVIII ${ }^{\mathrm{e}}$. Leurs auteurs étaient généralement des pasteurs protestants qui avaient pour mission d'éradiquer la religion indigène et de christianiser les Samis. Ils étaient totalement ignorants de la culture et de la religion de ces derniers et, à quelques exceptions près, ne parlaient pas leur langue. Leurs écrits n'avaient aucun but scientifique et leurs auteurs ne s'efforçaient pas de parvenir à des descriptions impartiales et nuancées. Ils cherchaient uniquement à améliorer leur connaissance de la religion indigène afin de mieux la combattre. Le matériel ainsi produit présente donc - pour le moins - certaines limites. Ces personnes étant intéressées par les questions théologiques, c'est la théologie qui déterminait la nature des questions qu'ils posaient aux Samis dans le cadre de leurs interrogatoires. Ils rassemblèrent ainsi une foule de détails sur les esprits qu'ils appelaient « les dieux ». Les textes rédigés pendant les années 1720 par les pasteurs influencés par le courant piétiste souffrent notamment du fait qu'ils mettent délibérément en avant les aspects de la religion indigène qui, d'un point de vue chrétien, étaient particulièrement odieux. Les Samis étaient souvent contraints, par la menace et la torture, de révéler ce qu'ils savaient de leurs propres rites et représentations religieuses. Mais, malgré les reproches qu'on peut leur adresser, ces écrits restent sans conteste nos plus importantes sources sur la religion indigène des Samis. Lorsque l'on songe à leur provenance, il est plutôt surprenant qu'ils nous donnent tant d'informations.

Si nous pouvons les utiliser - avec beaucoup de prudence et moyennant un examen critique sérieux - c'est que nous connaissons bien les idées des missionnaires et leurs méthodes de travail. L'orientation de ces écrits est claire, parfois même à l'excès, puisque la religion indigène est condamnée presque à chaque page. 
De ce qui vient d'être présenté, il ressort que le statut des sources diffère selon que l'on considère l'étude de la religion indigène chez les Samis ou bien chez de nombreux autres peuples autochtones, car la première se base sur des sources historiques. Cela veut dire que l'anthropologue qui étudie la religion indigène des Samis se place d'emblée dans une logique plus historique qu'anthropologique. Ce qui distingue le plus ces deux modes de travail est que les types de sources (documents historiques pour les uns et matériaux recueillis sur le terrain par les autres) déterminent dans quelle mesure il est possible de vérifier ou non les interprétations des chercheurs. Celui qui étudie le présent peut toujours contrôler auprès des représentants de la culture étudiée s'il l'a comprise ou non. Cette possibilité n'existe pas pour les travaux portant sur des périodes historiques révolues. Tout ce que l'on peut faire, c'est présenter une image compréhensible et cohérente de la religion étudiée, à partir du point de vue choisi, et à l'aide des matériaux disponibles. Dans ce cas, il est particulièrement important d'avoir conscience d'un fait qui s'applique à toute recherche culturelle : une autre perspective, reposant sur d'autres modèles théoriques, pourrait donner une autre image d'ensemble parce que le chercheur crée la religion (comme ensemble systématique) en «l'écrivant».

\section{RôLES SEXUELS}

Nous tenterons maintenant une synthèse de certains aspects de la religion indigène des Samis. Je me fonderai sur le matériel qui se rapporte aux Samis de la Laponie du sud pendant les premières décennies $d u X^{X}{ }^{e}{ }^{e}$ siècle : à la lumière d'une courte présentation des rôles sexuels qui étaient la norme à l'époque chez ces Samis, je résumerai ce que nous livrent les sources sur la conduite rituelle du nåjitie $^{5}$, que je considérerai comme un « jeu» plutôt que comme une « technique de l'extase », contrairement à ce qui a été fait jusqu'à présent. Pour définir encore plus précisément ma perspective, je me concentrerai sur les sources qui illustrent le « jeu érotique »,

5. Puisque cette analyse porte sur les Samis du sud, les mots cités seront transcrits selon l'orthographe du sami du sud. Ainsi, le mot nåejtie employé dans le sud correspond au mot noaidi en sami du nord. 
un thème qui n'a jamais été abordé jusqu'ici. Mais tout d'abord, quelques mots sur les rôles sexuels.

Dans la vie quotidienne, la division sexuelle du travail constituait une réalité évidente. Les hommes chassaient et pêchaient, gardaient les rennes et fabriquaient les skis, les traîneaux, les tentes, etc., tandis que, pour l'essentiel, les femmes élevaient les enfants, cousaient les vêtements, les chaussures, les gants, etc. C'était l'homme qui préparait les repas, alors que la femme trayait les animaux, fabriquait le fromage et lavait les ustensiles de cuisine.

Un missionnaire dénonçait même « le pouvoir et la tyrannie [des femmes samis] sur leurs maris »: la femme jouait effectivement un rôle dominant dans le foyer, et ce d'autant plus qu'elle était économiquement indépendante. Chez les gardiens de troupeaux, garçons et filles possédaient leurs propres rennes depuis leur petite enfance ${ }^{6}$.

Chez les adultes, le sexe constituait le critère essentiel de différenciation religieuse. L'homme comme la femme étaient soumis à des restrictions spécifiques en ce qui concernait les lieux et les objets sacrés pour l'autre sexe. Alors que les rites masculins portaient sur les aliments et les éléments, les rites féminins étaient liés au foyer et à la famille, par exemple les cérémonies de nomination. La religiosité de l'homme et celle de la femme entretenaient des rapports de contraste et de complémentarité mutuels. La division religieuse des rôles sexuels était stricte, mais pas totale; son caractère essentiel était la complémentarité et non la restriction.

Bien que cela transparaisse rarement dans les sources, on peut affirmer que les rôles des femmes et des hommes étaient particulièrement nets dans les rites sacrificiels. Dans la plupart des cas, il appartenait à l'homme d'effectuer les sacrifices d'animaux (surtout le renne), bien que les sources présentent ces sacrifices comme des actes collectifs indifférenciés effectués dans des lieux consacrés. Le sacrifice animal n'était pas, comme le laisse croire la littérature secondaire, un aspect de la religion des Samis, mais un aspect de la religion de l'élément masculin du peuple sami. Les sources ignorent presque complètement les offrandes de bouillon ou de

6. D'après H. Rydving, The End of Drum-Time, Religious Change among the Lule Saami, 1670s-1740s, Stockholm, Almqvist \& Wiksell International, (1993) 2004, p. 144-145. 
graisse faites par les femmes à l'intérieur du foyer, et la raison de cette ignorance réside dans le fait que les observateurs chrétiens étaient des hommes et n'avaient donc pas accès à l'univers religieux des femmes. Si cela reste compréhensible, il est davantage surprenant que la recherche ultérieure n'ait guère mis en évidence cette lacune, qui nous livre une image déformée de la religion des Samis?

La séparation des sphères religieuses respectives des hommes et des femmes se reflète également au niveau des esprits relevant de la religion sami. Bien que ces entités (les « dieux ») ne semblent pas avoir joué un rôle aussi prédominant dans la religion indigène que les textes le prétendent, un court tableau de la structuration du " monde des dieux » peut nous aider à comprendre encore mieux l'importance de la division du monde en une sphère masculine, une sphère féminine et une sphère commune aux deux sexes. Chez les Samis du sud, les déités féminines (dont les noms portaient les suffixes -aahka « femme, épouse », -iedne « mère » ou -niejte « fille ») étaient liées au commencement et à la fin de la vie ainsi qu'aux tâches et aux états de la femme, tandis que les déités masculines (dont les noms se terminaient entre autres par -gaellies «mari», -aehtjie « père » ou -ålmaj « homme ») régissaient le monde des hommes (la chasse, la pêche, l'activité pastorale). Cette division en « dieux » et « déesses » correspondait essentiellement à la répartition sexuelle des adorateurs des divinités respectives. L'exception la plus importante est la déesse de l'enfantement, Saaraahka, qui était vénérée par les deux sexes. Elle était le plus grand des esprits et fut même assimilée à la Vierge Marie. Plus précisément, on attribuait à Saaraahka le nom de Marie quand des prêtres ou d'autres chrétiens étaient à proximité, afin qu'ils ne sachent pas de qui l'on parlait. Le monogramme de Marie qui apparut sur le costume sami et les chants particuliers qui lui étaient adressés par des Samis de Lule (plus au nord) doivent être interprétés plutôt comme des messages codés ${ }^{8}$. Seuls les initiés savaient que c'était Saaraahka qui en était la destinataire?.

7. Rydving, The End of Drum-Time, p. 145-149.

8. H. Grundström, « Jungfru-Maria-motivet i lapska jojkningslåtar », Saga och sed, 1959, p. 52-57.

9. Rydving, The End of Drum-Time, p. 149-151. 


\section{RELATIONS RITUELLES}

\section{Les saajvh}

Les êtres invisibles (esprits) que les sources appellent « les dieux » occupent cependant (à l'exception de Saaraahka) une place moins importante que deux autres catégories d'êtres : les ancêtres défunts (plur. jaemegh) et les êtres souterrains que l'on appelait les saajvh. C'est à cette dernière catégorie que je vais m'intéresser pour analyser plus avant la conduite du nåejtie comme jeu, et en particulier comme jeu érotique.

Les Samis du sud croyaient que certaines montagnes étaient habitées par des esprits anthropomorphes intimement liés aux êtres humains, et qui entretenaient entre eux des relations familiales exactement comme les humains. Dans chacune de ces montagnes vivaient au moins un, et quelques fois plusieurs saajvh mâles (plur. saajveålmah), le plus souvent avec des saajvh femelles (plur. saajveniejth) et leurs petits. Les saajvh étaient la propriété des particuliers; on pouvait les vendre et les acheter, mais c'est surtout par voie d'héritage que les saajvh se transmettaient. Dans la société sami du sud, le nombre de saajvh d'un individu indiquait son statut social, mais en posséder beaucoup n'était pas nécessairement un avantage. Comme il était essentiel de maintenir l'équilibre entre l'élément masculin et l'élément féminin d'un couple, l'un ne devait pas avoir plus de saajvh que l'autre; sinon le conjoint qui en avait le plus devait vendre assez de « têtes » pour rétablir l'égalité des époux dans la famille ${ }^{10}$.

\section{Le nåejtie et les saajvh}

Dans la société sami préchrétienne, il y avait plusieurs types de spécialistes des rituels (hommes et femmes) qui assuraient la

10. H. Skanke, «Epitomes Historiæ Missionis Lapponicæ, Pars Prima anlangende de Nordske Lappers Hedendom og Superstitioner [1728-31]», dans O. Solberg (éd.), Nordlands og Troms finner i eldre håndskrifter, Oslo, Etnografisk museum, 1945, p. 190-194; cf. L. Bäckman, Sájva, Föreställningar om hjälp- och skyddsväsen $i$ heliga fjäll bland samerna, Stockholm, Almqvist \& Wiksell International, 1975; H. Rydving, "Synliga och osynliga landskap, Några samiska exempel », dans E. Mundal et A. Ågotnes (éd.), Ting og tekst, Bergen, Bryggens Museum, 2002, p. 71-75. 
communication avec les mondes habités par les ancêtres et les différents esprits. On faisait appel à eux en cas de maladie, on leur prêtait le pouvoir d'expliquer les phénomènes étranges, de prévoir l'avenir, et même de le modifier. Le plus important d'entre eux était le nåejtie. Lui aussi avait ses saajvh et ses saajvh anthropomorphes, que les historiens des religions appellent généralement les esprits gardiens. Les sources semblent nous permettre d'avancer que le nåejtie était considéré comme entretenant des liens particuliers avec au moins l'un de ses esprits gardiens féminins, relation qui avait même un caractère sexuel.

Si un futur nåejtie était la cible d'attaques de la part des esprits pour le contraindre à devenir nåejtie (et ces attaques duraient aussi longtemps qu'il hésitait), c'était l'un des esprits souterrains de sexe féminin (une saajveniejte) qui le fortifiait avec de l'eau ${ }^{11}$. Les sources n'en disent pas plus sur la saajveniejte, mais comme la plupart des Samis du sud que les missionnaires questionnaient étaient précisément des nåejtieh (plur. de nåejtie), il est permis de tenir compte des rapports sexuels que ces Samis disaient avoir eus avec leur saajvh et de supposer que les Samis incluaient également la sexualité dans les relations entre le nåejtie et son esprit gardien féminin (saajveniejte). Cette hypothèse est étayée par le caractère érotique du jeu rituel entre le nåejtie et la femme qui le secondait pendant les séances rituelles, et aussi par un mot (en sami de Nord) utilisé pour la totalité des esprits d'un nåejtie: snoallagazzi, «le troupeau qui parle de manière indécente ${ }^{12} »$.

\section{Chants érotiques et assistante rituelle}

Lorsque le nåejtie prenait part avec ses assistantes à ce que nous pouvons appeler des "jeux rituels", il entonnait un type de chant particulier (vuelie). Beaucoup de ces chants de nåejtie (nåejtievuelieh) avaient un contenu érotique, que la pruderie des auteurs de nos sources leur interdisait de rapporter. Mais des mots

11. J. Kildal, « Afguderiets Dempelse, og den Sande Lærdoms Fremgang [...] [1730] », dans M. Krekling (éd.), Nordlands og Troms finner i eldre håndskrifter, Oslo, Etnografisk museum, 1945, p. 139.

12. I. Olsen, «Om lappernes vildfarelser og overtro [après 1715]», dans J. Qvigstad (éd.), Kildeskrifter til den lappiske mythologi n ${ }^{2}$, Trondhjem, Det kgl. norske videnskabers selskabs, 1910, p. 46. 
tels que « obscénité » et « indécence » utilisés pour les juger nous en apprennent assez sur le caractère érotique des chants qui accompagnaient les jeux rituels. Ces renseignements sont un élément important du puzzle que forment les textes fragmentaires avec lesquels nous devons reconstituer le jeu rituel du nåejtie.

Interpréter les actes du nåejtie comme un jeu rituel nous permet de comprendre le rôle des aides mieux qu'on ne le ferait si, à la manière d'Eliade, on ne s'intéressait qu'à la question de savoir s'il était en «extase » ou non. En se plaçant dans notre perspective et en mettant en œuvre les sources disponibles pour reconstruire les activités rituelles du nåejtie et en tirer un système cohérent, il nous semble possible de présenter une forme-modèle du jeu rituel du nåejtie chez les Samis du sud de la manière suivante :

Le nåejtie se préparait par un jour de jeûne suivi de libations. Ensuite, il se mettait nu et commençait à jouer sur son tambour en chantant des vuelieh, accompagné par une femme ou une jeune fille non mariée, quelquefois par plusieurs femmes, qui chantaient avec lui. Pendant la partie du rite où le nåejtie, gisant immobile et muet sous la tente, était censé être parti vers les autres mondes, la femme continuait à chanter, racontant ce que faisait le nåejtie, quelles montagnes il traversait, qui il rencontrait, etc. C'est également elle qui, par son chant, le « réveillait » peu à peu ${ }^{13}$. Un récit concernant la côte septentrionale de la Laponie, dont l'auteur connaissait la langue sami ${ }^{14}$, nous en apprend davantage : à ce moment (du moins chez certains Samis du nord), le nåejtie chantait à son assistante plusieurs vuelieh érotiques, louant en particulier ses dons sexuels. Pour finir, il répondait aux questions que lui posait l'assistance sur les péripéties de son voyage.

Ces quelques éléments se présentent sous un jour nouveau si l'on interprète le comportement du nåejtie comme un jeu rituel, et parfois même comme un jeu érotique. On se rend compte alors du rôle central qu'occupait l'assistante dans cette dramaturgie. C'est elle qui raconte. C'est grâce à sa représentation que l'assistance avait connaissance des mondes invisibles que le nåejtie était censé avoir visité. Mais nous ne savons pas si le jeu rituel entre elle et le nåejtie

13. Skanke, « Epitomes Historiæ Missionis Lapponicæ, Pars Prima anlangende de Nordske Lappers Hedendom og Superstitioner [1728-31] », p. 205-209.

14. Olsen, « Om lappernes vildfarelser og overtro [après 1715] », p. 46-47. 
symbolise aussi la rencontre de celui-ci avec son esprit gardien (sa saajveniejte) dans le monde souterrain.

\section{Les esprits zoomorphes}

Outre les saajvh anthropomorphes, le nåejtie possédait (à la différence des autres membres de la communauté sami) un certain nombre d'esprits qui étaient à son service, entre autres plusieurs oiseaux. Les plus importants étaient trois esprits qui lui servaient d'assistants. C'étaient des esprits zoomorphes. Il y avait un oiseau (saajveledtie) qui aidait le nåejtie dans notre monde et dans l'audelà, et qui faisait aussi office de messager lorsque son maître voulait rencontrer l'un de ses saajvh anthropomorphes. Il y avait également un poisson (saajveguelie) qui l'assistait lorsqu'il rendait visite aux ancêtres dans le monde souterrain. Il y avait enfin un renne (saajvesaarva) qui assurait sa défense contre les autres nåejtieh et les esprits malveillants ${ }^{15}$. Aucune source ne permet d'interpréter dans un sens sexuel les rapports entre le nåejtie et ces entités, alors que c'est le cas pour certains spécialistes des rituels de Sibérie. Les seuls éléments qui pourraient encourager une telle interprétation sont quelques mots de la terminologie servant à décrire et caractériser les activités du nåejtie dans différents dialectes.

Si l'on considère l'ensemble du champ linguistique sami, on constate que la plupart des verbes se rapportant aux spécialistes des rituels font référence ${ }^{16}$ :

(a) soit à l'identité de l'officiant; par exemple, saN. noaiduhit et noaiddástallat indiquent qu'il s'agissait du noaidi ( $=$ saS. nåejtie), et saL. guobastallat qu'il s'agissait de la guobas (spécialiste féminine des rituels de la Laponie de Lule);

(b) soit aux instruments du rite; par exemple, saN. diiddastallat indique l'emploi de signes, saS. meevredh l'emploi du tambour;

(c) soit à l'objet du rite, comme dans ces quelques exemples tirés du sami du nord : geaidit « rendre quelqu'un invisible ou modifier son apparence physique », manidáhtit « chasser, par exemple une

15. Skanke, « Epitomes Historiæ Missionis Lapponicæ, Pars Prima anlangende de Nordske Lappers Hedendom og Superstitioner [1728-31] », p. 191-192.

16. $C f$. Rydving, "Shamanistic and postshamanistic terminologies in Saami », p. 191-197, où les mots sont transcrits avec un système orthographique antérieur. 
maladie », juovsahit « ramener un objet volé à son propriétaire », borrat « tuer» (en fait, c'est le mot usuel pour « manger »), etc.

Par ailleurs, $(d)$ quelques verbes sont employés pour caractériser les cris rituels du noaidi. Ce sont des mots que l'on emploie d'ordinaire pour nommer les cris des animaux, comme le verbe murret ("grogner comme un chien ») ou encore le verbe gihkat déjà cité, qui montre clairement que le rite était conçu comme un jeu érotique. Dans certains dialectes, ce dernier mot ne s'emploie que pour désigner le cri de certains animaux (notamment le grand coq de bruyère) pendant l'accouplement, tandis que dans d'autres dialectes il désigne également les cris rituels du noaidi. Dans les dialectes de Laponie orientale, il y a même une onomatopée spécifique pour désigner ce comportement: kharr-kharr ${ }^{17}$. Le mot gihkat est l'un des rares verbes dont nous savons qu'il était utilisé (du moins dans certaines régions) pour désigner la manière dont le noaidi procédait.

Ce verbe ne faisant pas partie du dialecte du sud, nous ne pouvons pas répondre avec certitude à la question de savoir si les nåejtieh chez les Samis du sud au début du XVIII' siècle imitaient le jeu amoureux de certains animaux. Mais si l'on tient compte du fait que, dans les autres régions samis, ils produisaient, comme les nåejtieh du sud pendant la célébration des rites, des cris rappelant ceux que le plus grand oiseau des forêts pousse pendant l'accouplement, le rapprochement avec les pratiques sibériennes ne peut être entièrement exclu.

\section{LA CONDUITE RITUELLE}

Quel était donc, concrètement, le schéma des rites accomplis par les noaiddit (nåejtieh, etc.)? En fait, nous savons très peu de choses sur ce thème, et cela est évidemment dû au fait qu'aucun des missionnaires chrétiens qui écrivirent sur les Samis n'eut jamais l'occasion d'assister à l'un de ces rituels. C'est pourquoi

17. T. I. Itkonen, Wörterbuch des Kolta- und Kolalappischen, Helsinki, Suomalais-ugrilainen seura, 1958, p. 37. 
les sources sont extrêmement allusives et généralement discrètes sur ce sujet. C'était très certainement des relations de troisième ou quatrième main ayant pour sources des Samis chrétiens qui avaient participé à de tels rituels avant leur conversion. L'une des plus complètes nous vient de Bergen, dans le sud de la Norvège, qui se trouve pourtant à une latitude bien inférieure à celle du territoire traditionnel des Samis. Mais Bergen était à cette époque le port de déchargement des produits vendus par les Samis (morue séchée, hareng et peaux), si bien que, suivant progressivement cet axe commercial nord/sud, ils finirent par atteindre cette ville. Quand la religion indigène était encore vivante, il arrivait qu'un noaidi vînt à Bergen, ce qu'atteste un court texte relatant un rituel qu'y conduisit l'un d'eux ${ }^{18}$.

Ce texte, un des rares documents pouvant prétendre au statut d'authenticité en matière de description de la conduite rituelle d'un noaidi ${ }^{19}$, fait partie de la littérature topographique nordique. Il s'agit du Norriges Oc Omliggende Øers sandfoerdige Bescriffuelse [...] (Description véridique de la Norvège et des îles environnantes) de Peder Claussøn Friis. Dans ce livre, publié en 1632, nous apprenons que deux hommes, qui n'étaient pas des Samis, furent témoins d'un rite conduit par un noaidi. Ils le virent se balancer, sauter en l'air, bondir à droite et à gauche, s'effondrer, rester immobile au sol puis se redresser. Le contexte dans lequel ce récit intervient indique qu'il avait pour but d'étayer la thèse de Friis, qui pensait que les Samis étaient restés des « païens » qui continuaient à se livrer à ce qu'il appelle «leur magie et adorations impies et effrayantes ${ }^{20} »$. Ce noaidi anonyme devait ainsi servir d'exemple édifiant destiné à conforter un stéréotype anti-ethnique. Cependant, Friis n'émet aucun jugement de valeur. Il s'abstient de tout commentaire moral ou religieux, se contentant de rapporter ce que les deux hommes avaient vu, un trait qui distingue ce texte de la plupart des récits conservés sur les rites samis.

Bien que la description que Friis fait d'un rite sami soit d'un

18. Plus détaillé dans H. Rydving, « Ein noaidi i Bergen », dans L. Mikaelsson (éd.), Religionsbyen Bergen, Bergen, Eide Forlag, 2000, p. 95-103.

19. Probablement du nord de la Laponie; j'écris par conséquent le mot en sami du nord.

20. P. C. [Friis], Norriges Oc Omliggende Øers sandfcerdige Bescriffuelse [...], Kiøbenhaffn, Melchior Martzan, 1632, p. 131. 
intérêt exceptionnel, elle n'est pas la seule; mais à la différence des autres textes, le témoignage rapporté par Friis ne fait mention ni de tambours ni d'assistantes. Il ne parle pas non plus de cris. Il ne donne pas d'information d'ordre auditif. Par contre, il insiste sur les détails visuels, un élément qui fait presque complètement défaut dans les autres témoignages qui nous sont parvenus :

Il se mit en place et se mit à se balancer comme s'il avait été ivre/et soudainement il bondit d'un trait/et sauta ici et là quelques fois/puis il s'effondra/et resta couché devant eux/comme mort/et lorsqu'il se fut peu à peu réveillé/il leur dit ce que les maîtres [de l'un des hommes] faisaient en Allemagne ${ }^{21}$.

C'est la brève description de la gestualité du noaidi qui confère à ce texte un caractère exceptionnel, et non l'objet de la séance.

Le déroulement gestuel du rite se divise en trois phases : mouvements (le noaidi se balance, bondit, saute, s'effondre), immobilité (il gît « comme mort»), reprise du mouvement (il se réveille). Ce que nos deux témoins avaient perçu comme une succession absurde de gestes était un comportement rituel acquis et précis. À travers un long enseignement, le futur noaidi apprenait à discipliner son corps en maîtrisant des figures gestuelles complexes. Les témoins n'observent que l'expression corporelle du noaidi. Ce texte illustre donc à merveille l'idée de Marcel Mauss sur les techniques corporelles comme résultat de l'imitation et de l'éducation ${ }^{22}$. Ce que faisait le noaidi était le produit d'un ordre établi par le ou les maîtres qu'il avait eus pendant le long processus d'apprentissage et d'initiation qu'il avait dû suivre. Dans ce cas précis, n'ayant pas accès au code culturel du noaidi, les deux étrangers ne pouvaient interpréter les significations symboliques de sa gestualité. Ils n'étaient que des témoins et non des participants. Bien qu'ils fussent présents dans le même local, ils se trouvaient dans un tout autre univers sémiotique et culturel. Dans le cadre normal du rite, c'était d'autres Samis qui observaient les gestes et l'immobilité du noaidi. Le rite était alors un acte collectif puisque le langage corporel du noaidi, son jeu rituel, s'adressait à des destinataires qui en comprenaient les significations. Dans la pièce de la maison de Bergen, il y avait un

21. [Friis], Norriges Oc Omliggende Øers sandfcerdige Bescriffuelse [...], p. 133.

22. M. Mauss, « Les techniques du corps [1936] », dans M. Mauss, Sociologie et anthropologie, Paris, PUF, 1980, p. 363-386. 
émetteur, mais aucun récepteur. Les messages émis par le corps du noaidi se perdaient dans le vide.

Dès lors, on conçoit facilement l'étonnement des deux témoins devant le spectacle qu'ils avaient sous les yeux. Dans le champ culturel du noaidi, le corps était intégré à l'activité rituelle tandis que, de plus en plus simplifiés, les rites de la liturgie luthérienne (dont nous pouvons supposer qu'ils étaient les seuls rites que connaissaient les témoins) constituent un exemple de la «désacralisation du corps » qui devait s'accentuer dans les siècles suivants. Nos deux témoins ont donc dû être complètement désarçonnés par les gestes du noaidi. Gesticulation chaotique pour eux, ces balancements, ce bondissement, cette chute, cette immobilité et ce redressement sont pour nous - quatre siècles plus tard - un ensemble gestuel parfaitement cohérent. Cependant, pas plus qu'eux, nous ne sommes capables d'en pénétrer le sens : ce savoir est mort avec la tradition du noaidi.

Le récit du rite effectué à Bergen par un noaidi dans les premières années du XVII ${ }^{e}$ siècle constitue un élément modeste mais non négligeable pour l'étude des fonctions centrales qu'assumait le noaidi dans la société sami préchrétienne. Nous ne savons pas comment ce noaidi est arrivé à Bergen, nous ne savons rien de son passé et rien de ce qu'il est devenu par la suite. Nous savons seulement ce qu'il a fait à l'occasion d'une rencontre particulière. Seul le récit d'une scène qui eut lieu dans une maison de Bergen conserve sur le papier un bref instant - guère plus d'une heure sans doute - de la vie du noaidi; tout le reste est depuis longtemps tombé dans l'oubli. Mais nous pouvons faire revivre une courte séquence d'actions, une mince tranche de temps, en suivant les lignes du texte de Friis. Nous pouvons discuter ce bref épisode et le mettre en relation avec d'autres acteurs décrits dans d'autres textes. Malgré le caractère fragmentaire de l'information qu'il nous livre, il est donc un exemple tout à fait précieux pour comprendre en quoi la conduite rituelle concrète du noaidi fonctionnait comme un jeu. Les deux témoins n'avaient pas la possibilité de saisir les aspects érotiques du jeu. Nous, en revanche, nous pouvons au moins émettre l'hypothèse que quelques-uns de ces gestes aient été une imitation du jeu amoureux de certains animaux. 


\section{RÉFLEXIONS FINALES}

Inspiré par l'analyse de Roberte Hamayon sur les rituels de certains peuples autochtones de Sibérie, j'ai présenté une lecture de quelques sources portant sur les rites des Samis. J'ai pu faire une synthèse nouvelle des renseignements disparates qu'elles nous livrent sur certains aspects des rites conduits par le noaidi (ou nåejtie). Les aspects érotiques du jeu rituel se sont révélés être une clé essentielle pour comprendre la raison des gestes et des cris du noaidi. Les informations que les sources livrent sur le jeu érotique entre le noaidi et son assistante; la condamnation par les missionnaires de certains chants; certains termes mettant les gestes du noaidi en rapport avec le jeu amoureux du plus grand oiseau de la forêt; un texte décrivant - quoique de manière fragmentaire - la gestuelle du noaidi : telles sont quelques-unes des pièces du puzzle. L'approche consistant à lire les sources du point de vue du « jeu rituel » s'est révélée féconde.

Mais comment allons-nous traiter les ressemblances entre la religion des Samis et les religions de quelques peuples sibériens, dans une discussion menée après la mort des Grandes théories? Autrefois, il aurait été facile de répondre à cette question en renvoyant, en fonction de ses propres présupposés, soit à une faculté universelle inhérente à l'être humain, par exemple le fonctionnement des processus cognitifs du cerveau humain (une théorie remise à l'honneur ces dernières années); soit à un héritage universel provenant d'une étape antérieure de l'évolution culturelle de l'être humain (selon la théorie du stade appelé « animiste », « totémique » ou « chamanique »); soit à un héritage universel par lequel «le sacré » se manifesterait sous un nombre déterminé de formes; soit à une évolution parallèle qui expliquerait par exemple que des conditions écologiques similaires susciteraient dans deux régions différentes des systèmes religieux analogues pour des cultures dites de « même niveau technologique "; soit à un héritage régional collectif formé d'un stratum culturel commun à l'ensemble (ou à certaines parties) de l'Eurasie septentrionale; soit à une théorie de la diffusion historique depuis la Sibérie vers l'ouest; soit enfin à une combinaison de deux ou davantage de ces explications, entre elles ou avec d'autres.

Tous ces modèles explicatifs ont eu (et certains ont encore) leurs adeptes qui avaient toujours réponse à tout. Nous autres, nous doutons; nous ne nous fions à aucune interprétation sans l'avoir 
soumise à l'épreuve de la probabilité (la macroperspective a fait place à la microperspective); nous pensons qu'il n'est pas convaincant d'apporter une même réponse à des questions différentes; nous évitons de tirer des conclusions générales, voire parfois de conclure quoi que ce soit; nous posons des questions car nous pensons qu'elles permettent parfois de mieux avancer sur la voie de la connaissance que certaines réponses.

Dans l'exemple présenté, les ressemblances sont des ressemblances heuristiques que la méthode nous fait découvrir; ce sont en effet précisément les ressemblances que nous cherchons dans nos sources à partir d'une approche reposant sur l'analyse du matériel d'un autre espace culturel. La méthode que j'ai illustrée est ainsi différente de la méthode comparative ou il s'agissait de comparer deux complexes de rites appartenant à deux (ou plusieurs) cultures, à partir d'une perspective déterminée. Dans cet essai, j'ai tenté de montrer qu'analyser un élément d'une religion à partir d'une autre, que lire un texte à partir d'un contexte qui lui est extérieur, peut être fécond. Mais les ressemblances et les analogies revêtent alors des fonctions et un statut théoriques autres que dans le cas des « vraies comparaisons »; elles illustrent surtout le fait que nos approches théoriques peuvent être perçues comme des démarches plus heuristiques qu'absolues. On comprendra facilement pourquoi la méthode que j'ai illustrée - qui consiste à mettre différentes interprétations à l'épreuve, à voir à quoi d'autres approches peuvent nous faire aboutir, à « jouer » avec des hypothèses - amène certains théoriciens à soutenir que même le processus de recherche devrait être considéré comme « un jeu ${ }^{23} »^{24}$.

hakan.rydving@ahkr.uib.no

\section{ABRÉVIATIONS}

saL. sami de la Laponie de Luleå

saN. sami du nord

saS. sami du sud

23. Gill, «Play », p. 459-460.

24. Je suis très reconnaissant à mon collègue Yves Delaporte (CNRS, Paris) pour la relecture du texte français. 


\section{BIBLIOGRAPHIE}

Louise Bäckman, Sájva, Föreställningar om hjälp- och skyddsväsen $i$ heliga fjäll bland samerna, Stockholm, Almqvist \& Wiksell International, 1975.

Gregory Bateson, Steps to an Ecology of Mind, New York, Ballantine, 1972.

Roger CAillois, Les jeux et les hommes, Paris, Gallimard, [1958] 2000.

Peder Claussøn [FrIIS], Norriges Oc Omliggende Øers sandfcerdige Bescriffuelse [...], Kiøbenhaffn, Melchior Martzan, 1632.

Sam D. GiLl, « Play », dans W. Braun et R. T. McCutcheon (éd.), Guide to the Study of Religion, London, Cassell, 2000, p. 451-462.

Harald GRUNDSTRÖM, « Jungfru-Maria-motivet i lapska jojkningslåtar », Saga och sed, Upsal, Kungl. Gustav Adolfs Akademien, 1959, p. 52-57.

Roberte N. Hamayon, « Stakes of the game, Life and death in Siberian shamanism ", Diogène : revue internationale de sciences humaines, $\mathrm{n}^{\mathrm{o}} 158,1992$, p. 69-85.

Roberte N. HAMAYON, « Pourquoi les “jeux” plaisent aux esprits et déplaisent à Dieu. Le "jeu", forme élémentaire de rituel à partir d'exemples chamaniques sibériens ", dans G. Thinès et L. de Heusch (éd.), Rites et ritualisation, Paris, Librairie philosophique J. Vrin, 1995, p. 65-100.

Roberte N. Hamayon, « Pour en finir avec la « transe » et l' « extase » dans l'étude du chamanisme », Études mongoles et sibériennes, n 26, 1995, p. 155-190.

Roberte N. Hamayon, « L'épingle du jeu », La Revue du MAUSS, n 12 , 1998, p. 103-121.

Roberte N. Hamayon, «Des usages de 'jeu' dans le vocabulaire rituel du monde altaïque », Études mongoles et sibériennes, $\mathrm{n}^{\circ}$ 30-31, 2000, p. 11-45.

Johan Huizinga, Homo ludens, Essai sur la fonction sociale du jeu, Paris, Gallimard (1938) 1988.

Toivi I. ItKonen, Wörterbuch des Kolta- und Kolalappischen, Helsinki, Suomalais-ugrilainen seura, 1958.

Jens KILDAL, « Afguderiets Dempelse, og den Sande Lærdoms Fremgang [...] [1730] », dans M. Krekling (éd.), Nordlands og Troms finner i eldre håndskrifter, Oslo, Etnografisk museum, 1945, p. 102-152. 
Marcel Mauss, « Les techniques du corps [1936] », dans M. Mauss, Sociologie et anthropologie, Paris, PUF, 1980, p. 363-386.

Isaac Olsen, " Om lappernes vildfarelser og overtro [après 1715] », dans J. Qvigstad (éd.), Kildeskrifter til den lappiske mythologi, $\mathrm{n}^{\circ} 2$, Trondhjem, Det kgl. norske videnskabers selskabs, 1910, p. 1-101.

Håkan Rydving, "Shamanistic and postshamanistic terminologies in Saami », dans T. Ahlbäck (éd.), Saami Religion, Stockholm, Almqvist \& Wiksell International, 1987, p. 185-207.

Håkan Rydving, Samisk religionshistoria, Några källkritiska problem, Stockholm, Almqvist \& Wiksell International, 1995.

Håkan Rydving, «The missionary accounts from the 17th and 18th centuries, The evaluation and interpretation of the sources », dans J. Pentikäinen (éd.), Sami Folkloristics, Turku, Nordic Network of Folklore, 2000, p. 17-39.

Håkan Rydving, «Ein noaidi i Bergen », dans L. Mikaelsson (éd.), Religionsbyen Bergen, Bergen, Eide forlag, 2000, p. 95-103.

Håkan Rydving, « Synliga och osynliga landskap, Några samiska exempel », dans E. Mundal \& A. Ågotnes (éd.), Ting og tekst, Bergen, Bryggens Museum, 2002, p. 65-77.

Håkan Rydving, The End of Drum-Time, Religious Change among the Lule Saami, 1670s-1740s, Stockholm, Almqvist \& Wiksell International, [1993] 2004.

Hans Skanke, « Epitomes Historiæ Missionis Lapponicæ, Pars Prima anlangende de Nordske Lappers Hedendom og Superstitioner [1728-31] », dans O. Solberg (éd.), Nordlands og Troms finner $i$ eldre håndskrifter, Oslo, Etnografisk museum, 1945, p. 179-224. 